\title{
Philosophiques
}

\section{La seconde théorie du langage de Wittgenstein}

\section{Denis Sauvé}

Volume 22, numéro 2, automne 1995

URI : https://id.erudit.org/iderudit/027329ar

DOI : https://doi.org/10.7202/027329ar

Aller au sommaire du numéro

Éditeur(s)

Société de philosophie du Québec

ISSN

0316-2923 (imprimé)

1492-1391 (numérique)

Découvrir la revue

Citer cet article

Sauvé, D. (1995). La seconde théorie du langage de Wittgenstein. Philosophiques, 22(2), 213-236. https://doi.org/10.7202/027329ar

\section{Résumé de l'article}

Les remarques de Wittgenstein sur le langage dans les Recherches philosophiques contiennent-elles une " théorie » du langage ? Je défends l'interprétation d'après laquelle Wittgenstein avance une théorie du langage ou au moins l'ébauche d'une telle théorie. Ses deux principales composantes (la première relative à la nature du langage, la seconde à sa structure) est, d'une part, la théorie de la "signification-usage » et, de l'autre, l'idée suivant laquelle les langues naturelles sont des " agglomérats » ou des « mélanges » de jeux de langage plus ou moins similaires aux formes de langage simples décrites au début des Recherches. 


\title{
LA SECONDE THÉORIE DU LANGAGE DE WITTGENSTEEN
}

\author{
par \\ Denis Sauvé
}

\begin{abstract}
RÉSUMÉ : Les remarques de Wittgenstein sur le langage dans les Recherches philosophiques contiennent-elles une "théorie " du langage? Je défends l'interprétation d'après laquelle Wittgenstein avance une théorie du langage ou au moins l'ébauche d'une telle théorie. Ses deux principales composantes (la première relative à la nature du langage, la seconde à sa structure) est, d'une part, la théorie de la " signification-usage » et, de l'autre, l'idée suivant laquelle les langues naturelles sont des "agglomérats "ou des "mélanges" de jeux de langage plus ou moins similaires aux formes de langage simples décrites au début des Recherches.

ABSTRACT:Do Wittgenstein's remarks on language in the Philosophical Investigations amount to $a$ " theory " of language? I defend the view that Wittgenstein does have a theory of language or the sketch of such a theory. Its two main components (pertaining to the nature and to the structure of languages) are the "use-theory of meaning " and the idea that natural languages are "conglomerates" or "mixtures " of language-games more or less similar to the simple forms of language he describes in the Investigations.
\end{abstract}

Pour beaucoup de ses interprètes, on ne doit pas chercher dans les Recherches philosophiques de Wittgenstein une théorie du langage ${ }^{1}$. Je défen-

1. Cf. par exemple G. E. M. Anscombe, « A Theory of Language? », dans I. Block (éd.), Perspectives on the Philosophy of Wittgenstein, Cambridge, Mass., MIT Press, 1981, p. 148-158 ; J. Bogen, Wittgenstein's Philosophy of Language, Londres, Routledge and Kegan Paul, 1972, p. 199-218; et W. D. Goldfarb, « I Want You to Bring Me a Slab : Remarks on the Opening Sections of the Philosophical Investigations 》 (Synthese, vol. 56, 1983), p. 265-282. Je fais plus loin quelques remarques critiques sur les interprétations de Bogen et de Goldfarb (cf. section 5). Anscombe, si je comprends bien, tente de montrer qu'il n'y a pas de théorie du langage dans les Recherches en essayant de réfuter une version particulière de la théorie qu'elle expose de façon extrêmement sommaire au début de son article. On pourrait lui objecter que cette version de la théorie n'est peut-être pas celle qu' accepterait Wittgenstein ou, en admettant que son interprétation est correcte, 
drai l'interprétation opposée. Je pense que l'on trouve dans les écrits du second Wittgenstein une « théorie du symbolisme », une théorie - ou du moins l'esquisse d'une théorie - qui propose de dire (entre autres) en quoi consiste la signification d'une expression dans une langue 2 .

On pourrait objecter qu'il écrit dans les Recherches : «[...] nos considérations ne [peuvent] être scientifiques. [...] Et nous ne pouvons avancer aucune espèce de théorie. Il ne doit rien y avoir d'hypothétique dans nos considérations ${ }^{3}$. ». Mais il semble vouloir plutôt rejeter à cet endroit l'idée d'une théorie explicative (causale) du langage : il pourrait estimer qu'une théorie du langage est possible mais soutenir que l'essence du langage est indépendante des mécanismes qui expliquent causalement les comportements linguistiques des locuteurs (un peu comme le fonctionnalisme en philosophie de la psychologie qui suppose que la nature d'un état mental est indépendante de ses "réalisations" physiques").

Une autre objection pourrait être qu'il nie que le langage ait une essence. Il écrit par exemple : "Au lieu d'indiquer ce qu'il y a de commun à tout ce que nous appelons un langage, je dis qu'il n'y a pas une seule chose qui soit commune à tous ces phénomènes [...] mais que [ceux-ci] sont apparentés les uns aux autres de bien des façons différentes ${ }^{5}$. » Wittgenstein fait allusion au point de vue qu'il défendait dans le Tractatus suivant lequel tous les usages du langage ont une fonction " référentielle " et " descriptive ". Il défend maintenant l'idée qu'il y a d'autres « usages " du langage que ceux-là. Mais il ne semble pas qu'il veuille dire également que les expressions d'une langue n'ont pas au moins la propriété commune d'avoir une signification. Il est toutefois possible que, pour lui, le langage n'ait pas d' « essence » non pas parce que le fait d'avoir une signification n'est pas un trait commun à toute forme de langage mais parce qu' autre chose qu'un signe (mot, expression ou phrase) pourrait être doué de signification (par exemple une pièce d'un jeu d'échecs). Je supposerai dans ce qui suit que seuls les signes (linguistiques) ont selon lui une signification et je reviendrai brièvement à la fin sur ce point.

que sa réfutation de la théorie ne prouve pas qu'il ne serait pas d'accord avec la théorie en question.

2. Les abréviations pour les références aux écrits de Wittgenstein seront les suivantes. $P U$ pour Philosophische Untersuchungen (I) G. E. M. Anscombe et R. Rhees (éd.), Francfort, Suhrkamp, $1969 ; B B$ pour The Blue and Brown Books, Oxford, B. Blackwell, $2^{\mathrm{e}}$ édition, $1969 ; Z$ pour Zettel, G. E. M. Anscombe et G. H. von Wright (éd.), Berkeley et Los Angeles, University of California Press, 1967 ; PG pour Philosophische Grammatik, R. Rhees (éd.), Francfort, Suhrkamp, 1969 ; et WLC pour Wittgenstein's Lectures, Cambridge 1932-1935 (From the Notes of A. Ambrose and M. Macdonald), A. Ambrose (éd.), Chicago, University of Chicago Press, 1982.

3. $P U, \S 109$.

4. Cf. $P U, \S 108$.

5. $P U, \S 65$. 
Je pense que les deux affirmations les plus importantes de Wittgenstein au sujet du langage sont les suivantes: (i) un signe (un son ou une inscription) n'a une signification, un certain contenu sémantique, que dans des « jeux de langage "; autrement dit, la signification d'une expression est fonction de l' " usage » qu'en font les locuteurs dans ces jeux. (ii) Le langage de tous les jours - une langue naturelle comme le français - est une collection ou une " famille $»^{6}$ de jeux de langage.

Je procéderai comme suit. J'examinerai d'abord ce qu'il entend par l'expression de « jeu de langage " et quels problèmes cela soulève (section 1). J'expliquerai ensuite en quel sens la signification est l' " usage » (section 2). Wittgenstein invoque pour illustrer (i) des exemples de jeux de langage " simples » comme ceux décrits au début des Recherches ou dans la première partie du Cahier brun (la thèse [i] fait l'objet grosso modo des vingt-cinq premiers paragraphes des Recherches). Après cela, je traiterai de la question de savoir si (i) est vraie également du langage de tous les jours, c'est-à-dire si, comme l'affirme (ii), les langues naturelles sont des collections de jeux de langage (section 3) et si la signification des expressions et des énoncés dans une langue naturelle est fonction de l' " usage " qu'en font les locuteurs dans ces jeux (section 4). Je répondrai pour terminer à des objections que l'on pourrait opposer à mon interprétation (section 5).

\section{Qu'est-ce qu'un jeu de langage ?}

Un jeu de langage se définit en premier lieu comme un «système de communication » plus « simple » ou « primitif » que les systèmes de communication beaucoup plus « développés " 7 et « complexes » que représentent les langues naturelles. Des exemples de jeux de langage seraient les premières formes de langage des enfants ou les systèmes de signes employés dans des groupes humains primitifs. Dans un jeu de langage, dit par ailleurs Wittgenstein, s'entre-mêlent l'emploi de signes et les actions qui leur sont liées : "l'expression "jeu de langage" doit mettre [...] en valeur le fait que parler un langage est une partie d'une activité ou d'une forme de $v^{8}{ }^{8}$ ".

Un exemple de jeu de langage serait le jeu du constructeur ${ }^{9}$. Un " constructeur » (A) utilise des matériaux tels que des briques, des dalles ou des poutres. Il donne parfois des ordres à son aide (B) qui les exécute. Les seuls mots employés sont «poutre », « brique », « dalle », etc. A demande à B d'apporter une poutre : il prononce le mot « Poutre! " et B obéit. Une activité dans le jeu associée à l'usage des signes est l'action d'exécuter les ordres. «Concevez [ce jeu de langage], dit Wittgenstein, comme un langage primitif

\footnotetext{
6. Cf. $P U, \S 108$.

7. $C f . P G$, p. 62 .

8. $P U, \S 32 ; c f . P U, \S 7$.

9. Cf. $P U, \S 2$.
} 
complet $^{10}$. " Il est complet en ce sens que rien ne manque au jeu pour qu'on puisse dire qu'il s'agit d'un langage (ou d'une « forme de langage »). On peut imaginer une "extension $"{ }^{11}$ du jeu qui comporte d'autres types de mots et leurs règles d'emploi mais le nouveau jeu ne serait pas plus « complet » qu'il ne l'était auparavant - on aurait simplement inventé un autre jeu ${ }^{12}$. De plus, « on pourrait imaginer que ce jeu de langage soit tout le langage de A et de B, voire tout le langage d'une tribu [primitive] ${ }^{13}$ ».

Des commentateurs ont objecté que le jeu de langage du constructeur n'est pas ce qu'on appelle ordinairement un jeu. Pour Max Black, un jeu doit avoir les propriétés suivantes ${ }^{14}$ : (1) sa raison d'être est le divertissement ; (2) il est régi par des règles ; (3) il est dirigé vers un but (qui met fin au jeu) ; (4) l'atteinte du but n'a aucune « valeur intrinsèque » (jouer n'est pas une activité «sérieuse » ou «utile »); (5) les règles sont arbitraires; et (6) il est "délimité » : son état initial et son état final sont fixés par une convention. Un " test décisif » pour qu'une activité soit correctement appelée un « jeu », dit Black, est la satisfaction du critère (1). Le jeu des constructeurs, comme la plupart des autres exemples des Recherches, ne sont pas des jeux dans la mesure où ils n'ont pas pour but le divertissement (je suppose que (4) ou peut-être (6) mènent à la même conclusion).

Pour Wittgenstein, il y a des similitudes intéressantes entre jeux et langage (il est question à un endroit dans les Recherches de l' " analogie » entre jeux et langage $)^{15}:$ il ne dit pas que les jeux de langage sont littéralement des jeux. On peut mentionner les six propriétés suivantes : (1) le caractère multiforme des jeux de langage (il y a une grande variété de jeux : les échecs, le Scrabble, les jeux qu' on joue avec des cartes, etc.) ; (2) le fait qu'on trouve seulement des « ressemblances de familles» entre différents jeux de langage (lesquels n'ont pas par exemple seulement un usage « référentiel » ou " descriptif »); (3) un jeu de langage comporte toutes sortes d'activités outre l'usage de signes impliquant souvent plusieurs participants (c'est le cas également des jeux : jouer une partie de cartes ne consiste pas uniquement à manipuler des cartes mais à effectuer des actions telles que prononcer des mots, inscrire des résultats, etc.) ; (4) comme les jeux, les jeux de langage sont " complets" (ajouter ou retirer des pièces du jeu d'échecs ne le rendrait ni plus ni moins « complet»); (5) les règles d'emploi d'un langage, comme les règles des jeux, sont arbitraires ( La grammaire n'a de comptes à rendre à aucune réalité $\left.{ }^{16} . »\right) ;(6)$ enfin, les jeux de langage, comme les jeux, sont des

10. $P U, \S 2$.

11. $C f . P U, \S 8$.

12. $C f$. aussi $P U, \S 18$ et $W L C$, p. 101-102.

13. $P U, \S 6$.

14. M. Black, «Wittgenstein's Language-Games » dans S. G. Shanker (éd.), Ludwig Wittgenstein : Critical Assessments, Beckenham, Croom Helm, 1986, p. 82-83.

15. $C f . P U, \S 83$.

16. $P G$, p. 184 . 
pratiques sociales, des formes réglées d'interaction entre individus. On peut voir d'autres similitudes entre jeux et langage, mais celles-là semblent les plus importantes.

La difficulté principale dans le concept d'un jeu de langage, pour beaucoup de commentateurs, tient à ce qu'un jeu de langage est censé être "complet » et qu'il pourrait être le seul langage parlé par des êtres humains ${ }^{17}$. Max Black écrit : «il est effectivement concevable [...] que l'on découvre une tribu où les gens [...] ne jouent aucun autre jeu [que les échecs]. " Mais « une thèse parallele à propos des jeux de langage se heurte à de sérieuses difficultés ${ }^{18}{ }^{»}$. Pour Rush Rhees, la difficulté est « d'imaginer que [les constructeurs] ne parlent [ce] langage que pour donner des ordres, en exécutant ce genre de travail, et autrement ne parlent pas du tout ${ }^{19}$ ". " Je ne pense pas, dit Rhees, que ce serait parler un langage ${ }^{20}$. » En effet, les mots («poutre », « dalle», etc.) «ne peuvent être employés d'aucune autre façon » ou dans d'autres genres de circonstances que celles dans lesquelles ils sont utilisés comme ce serait le cas $s^{\prime} i l$ s'agissait d'un véritable langage ${ }^{21}$. De plus, quand $A$ et $B$ ne travaillent pas (lorsque, disons, ils sont en train de cultiver leurs jardins), ils ne communiquent pas entre eux verbalement (ils n'ont pas dans leur vocabulaire des mots tels que "plante », « terre », " pluie », etc.). D'autre part, comme le note Black, A et B vont parfois avoir besoin " de communiquer des désirs, des intentions, de rapporter des états de choses et ainsi de suite, [ce qui] supposerait d'autres mots ou, à tout le moins, des mouvements de tête expressifs, des sourires et autres gestes appartenant à d'autres jeux de langage ${ }^{2}$ ». Par ailleurs, comme l'a fait remarquer entre autres Anthony Kenny ${ }^{23}$, les enoncés du constructeur sont dénués de structure. Ils font penser, dit Rhees, à de simples «signaux $»^{24}$. On est Ioin, d'apress Black, d'un exemple d' « authentique communication humaine ${ }^{25} "$.

En ce qui concerne le point que soulève Kenny, je pense que la réponse de Wittgenstein serait que les énoncés « Poutre !» dans le langage des constructeurs et «Apporte-moi une poutre ! » en français ont la même signification dans la mesure où il ont approximativement le même usage dans leurs langages respectifs ${ }^{26}$. Un énoncé comme "Poutre! " peut avoir une signification sans être syntaxiquement complexe. S'il a une signification — la même

17. Cf. aussi $B B$, p. 81 .

18. M. Black, « Wittgenstein's Language-Games », p. 79.

19. R. Rhees, «Wittgenstein's Builders », dans ses Discussions of Wittgenstein, Londres, Routledge and Kegan Paul, 1970, p. 76.

20. Ibid., p. 76.

21. Ibid., p. 76.

22. M. Black, «Wittgenstein's Language-Games », p. 79.

23. A. Kenny, Wittgenstein, Londres, Allen Lane, The Penguin Press, 1973, p. 169.

24. R. Rhees, “Wittgenstein's Builders », p. 77.

25. M. Black, «Wittgenstein's Language-Games », p. 81.

26. $C f . P U, \S 20$. 
que celle de son équivalent français - il semble que le jeu soit effectivement une forme de langage (je reviendrai sur ce point).

Norman Malcolm a essayé de répondre à l'objection de Rhees (qui pense qu'il n'est pas possible que des individus emploient un langage seulement dans ce genre de situation). Malcolm imagine une société primitive dans laquelle les activités et le type de milieu sont tels que les individus n' ont besoin d'aucun autre langage (ou d'un langage plus complexe) que celui du constructeur. Par exemple, dit Malcolm, « ils ne possèdent aucun mot pour [désigner] la nourriture parce que la nature leur en fournit en abondance et à leur portée ; aucune planification ou préparation n'est nécessaire ${ }^{27} \%$. D'autre part, « [ils] n'avertissent pas autrui lorsqu'il y a un danger [ils n'ont pas un jeu dans lequel un individu en avertit un autre] ; mais si l'un d'eux est attaqué par un animal sauvage, les autres vont venir à son aide ${ }^{28}$ ". Et ainsi de suite. On pourrait objecter que cela suppose qu'ils possèdent d'autres formes de communication : ils doivent être capables de communiquer au moyen de gestes, de mimiques, etc. Comme le note Black, $A$ et $B$ doivent pouvoir communiquer d'autres désirs, intentions ou croyances (B peut vouloir signaler à A qu'il n'a pas trouvé l'objet demandé, qu'il ne veut plus travailler, etc.).

Wittgenstein (ou Malcolm) ne répond pas à l'objection et il est difficile de voir comment on peut y répondre. La vie en société suppose différentes formes de coopération entre individus (ententes, tâches communes, échanges de biens, etc.) et au moins une certaine variété de formes (peut-être "primitives ») de langage de sorte que, comme le fait remarquer Black, il n'est pas plausible de supposer que A et $B$ ne jouent aucun autre jeu de langage (bien qu'on puisse imaginer qu'ils ne jouent pas d'autres jeux que les échecs). Je pense que Wittgenstein pourrait concéder que c'est le cas, mais maintenir que les jeux de langage sont " complets » et indépendants des autres jeux et que chacun constitue un langage.

Pour Rhees et beaucoup d'autres interprètes des Recherches, l'affirmation suivant laquelle le jeu du constructeur est un langage a quelque chose de contre-intuitif. En quel sens est-ce un langage ? Au sens où les signes utilisés ont une signification (ce sont des "symboles"). On pourrait imaginer un langage dans lequel il n'y aurait qu'un signe (par exemple "poutre " comme dans le jeu du constructeur) : si celui-ci a une signification, on est déjà en présence d'un langage. Il suffit (et il est nécessaire), pour que le signe ait une signification, qu'il ait un usage dans un jeu de langage. Le concept de jeu de langage, je pense, est introduit au début des Recherches dans le but (entre autres) d'élucider le concept de signification.

27. N. Malcolm, « Language-Game (2)», D. Z. Phillips et P. Winch (éd.), Wittgenstein : Attention to Particulars, Essays in Honor of Rush Rhees, New York, St. Martin's Press, 1989, p. 43.

28. Ibid., p. 44 


\section{2. « La signification est l'usage »}

"Pour une grande part des cas d'emploi du mot "signification" [...]", comme on lit dans un texte souvent cité des Recherches, " on peut l'expliquer ainsi : la signification d'un mot est son usage dans le langage ${ }^{29} »$. Wittgenstein avance à cet endroit et dans les paragraphes qui le précedent ce que l'on pourrait appeler sa théorie de la « signification-usage ». Mais en quoi consiste l'« usage » d'un mot?

On parle souvent dans le langage courant de l' « usage » (ou « emploi ») d'un mot (expression ou phrase) ${ }^{30}$. Quelqu'un peut dire : «Le conférencier a employé douze fois le mot "cacophonie" dans son exposé " ou bien : "Pour affirmer que la neige est blanche, on fait normalement usage du mot "neige". » Mais des remarques comme celles-là ne nous renseignent pas sur la signification de " cacophonie " ou de " neige".

J. F. M. Hunter propose l'interprétation suivante : le type de contexte auquel pense Wittgenstein est plus précisément celui où quelqu'un explique la signification d'un mot ou d'une expression. Quand le verbe «signifier», dit Hunter, est employé "soit lorsque nous expliquons la signification d'un mot, soit lorsque nous demandons une telle explication [...], au lieu de "signifie" nous pouvons écrire "a le même usage que" ou bien nous pouvons remplacer "signification" par "usage",31 ». Au lieu de " "Célibataire" signifie "personne non mariée" ", on pourrait dire : "Célibataire" a le même usage que "personne non mariée" "; et au lieu de " "Célibataire" et "personne non mariée" ont la même signification », on aurait : «"Célibataire" et "personne non mariée" ont le même usage ".

Le problème est qu'il n'y a apparemment rien d'étrange à dire également dans le langage courant des choses telles que "En français, le mot "pomme" et le mot "poire" ont le même usage. » On pourrait vouloir dire entre autres que les contextes dans lesquels on emploie habituellement les deux mots sont similaires (on dit «manger une poire » et « manger une pomme », " couper en deux une poire " et " couper en deux une pomme", etc.) ; que les pommes et les poires appartiennent à la même classe de choses (par opposition, disons, aux figures géométriques ou aux nombres); ou que, du point de vue de la grammaire, les comportements de " pomme " et de "poire " sont similaires (et diffèrent de ceux d'un verbe, d'un article ou d'un adjectif). On pourrait imaginer d'autre part que, à quelqu'un qui déclare «On fait en français le même usage de "célibataire" et de "personne non mariée" ", un auditeur ne connaissant pas la signification de ces expressions demande « En quel sens le même usage ? Au sens auquel on peut les employer comme sujets d'une

29. $P U, \S 43$.

30. Cf. W. P. Alston, « Meaning and Use », G. H. R. Parkinson (éd.), The Theory of Meaning, Oxford, Oxford University Press, 1968, p. 143-145.

31. J. F. M. Hunter, "Wittgenstein on Meaning and Use », E. D. Klemke (éd.), Essays on Wittgenstein, Urbana, University of Illinois Press, 1971, p. 382. 
phrase ou auquel elles sont partout interchangeables ou bien en un autre sens ? " La réponse serait probablement : "Elles ont le même usage en ce sens qu'elles ont la même signification. " .

Le concept d'usage est apparemment trop vague (ou trop général ?) pour expliquer celui de signification. Mais avant de tirer cette conclusion, je crois qu'on doit se demander, ce que ne fait pas Hunter, en quoi consiste l' « usage " d'un mot (expression ou phrase). Il faut tenir compte d'autres passages que le seul paragraphe 43 des Recherches auquel il se réfere ; je citerai entre autres des passages du Cahier brun dans lequel Wittgenstein est souvent beaucoup plus explicite que dans les Recherches.

Wittgenstein demande à un endroit dans les Recherches : comment une flèche peinte sur un panneau indicateur peut-elle indiquer une direction? Il écrit : « [La fleche] ne semble-t-elle pas porter déjà en elle-même quelque chose qui lui est extérieur [à savoir ce qu'elle est indique] ? - "Non, ce n'est pas la ligne morte [sur le panneau qui indique une direction] ; seul ce qui est psychique [das Psychische], la signification, peut faire cela." " Il ajoute pour commenter la réponse : " C'est à la fois vrai et faux. La flèche n' indique que dans l'application [Anwendung] qu'en fait un être vivant. Cet acte d'indiquer [dieses Zeigen] n'est pas un tour de passe-passe que seule l'âme peut effectuer $^{32}$ ".

Wittgenstein s'attaque à plusieurs endroits à une conception «mentaliste » du langage. La signification, suivant cette conception, est quelque chose qui est présent (ou se passe) dans nos esprits. Elle est par exemple l' " idée " (ou " idée générale " ${ }^{33}$ ) qu' on associe à un mot, un processus dans l'esprit ou une image mentale. Ainsi, pour Frege, ce qui "donne vie " aux signes (ce qui doit « s'ajouter » au son pour qu'il soit « vivant » et ait une signification) est le « sens » ou la " pensée »; Wittgenstein écrit : « [s]ans un sens, ou sans la pensée [selon Frege], une proposition serait un chose totalement inerte et dénuée d'intérêt ${ }^{34} "$. De façon similaire, on pourrait être tenté de dire que la flèche n'indique une direction que si elle est interprétée de cette manière par ceux qui la voient, c'est-à-dire si quelque chose se passe " dans leur esprit » «signifiant » que la flèche pointe effectivement dans cette direction. Mais la signification au contraire n'est pas quelque chose qui se situe (ou a lieu) « dans nos esprits » : la flèche n'indique une direction que dans son " application » ou son " usage ». Supposons que quelqu'un s'arrête à une intersection et hésite sur le chemin à emprunter : il aperçoit un panneau sur lequel on a dessiné une flèche ; il regarde dans la direction qu' indique la flèche et se met à marcher dans cette direction. La flèche indique ("signifie") quelque chose parce que c'est en cela, entre autres, que consiste son $"$ usage ${ }^{35}$.

32. $P U, \S 454$.

33. Cf. $W L C$, p. 77.

34. $B B$, p. 4.

35. Cf. $P U, \S 432$ : «Chaque signe isolé [allein] semble mort. Qu'est-ce qui lui donne 
On trouve d'autres exemples d' " usages" (en ce sens particulier à Wittgenstein) dans un jeu de langage du Cahier brun où des individus emploient différents types de « noms ». L'un (A) demande à un autre (B) d'aller faire des achats. A remet à $B$ un bout de papier sur lequel il a griffonné quatre signes. Le premier est le nom d'un magasin: $\mathrm{B}$ a été entraîné à se rendre au magasin sur la porte duquel on a peint le signe. B s'y rend, entre et prononce un son correspondant au deuxième signe ; un des employés vient alors vers lui (l'employé a été entraîné à accourir chaque fois qu'il entend le son) : ce deuxième type de signe est un nom de personne. B montre alors à l'employé le troisième et le quatrième signe. On lui a enseigné à associer au premier des deux signes un certain type d'objet, disons des pommes (ce sont des noms de choses) et, quand l'employé auquel B s'adresse voit l'autre signe (un nom de nombre), il va chercher une certaine quantité d'objets correspondant au troisième (ici cinq pommes) et il vient les remettre à $\mathrm{B}$.

En quoi consiste la relation entre le nom et l'objet ou type d'objet qu'il désigne, par exemple entre le nom d'une maison et la maison? On peut donner deux genres de réponses :

La première est que la relation consiste en ce que certains traits ont été peints sur la porte de la maison. La deuxième [...] est que la relation qui nous intéresse est établie non pas seulement par le fait qu'on a peint ces traits sur la porte mais par le rôle particulier que [ceux-ci] jouent dans la pratique de notre langage telle que nous l'avons esquissée. - De même, ici, la relation du nom d'une personne à cette personne consiste en ce que la personne a été entrânée à accourir vers quelqu'un qui l'a appelée en prononçant son nom ; ou bien encore nous pourrions dire que [la relation] consiste en cela ainsi que dans la totalité de l'usage du nom dans le jeu de langage ${ }^{36}$

Un signe inscrit sur un bout de papier est le nom d'une personne en vertu de la totalité de son usage, aussi bien celui fait par ceux qui l'écrivent (ou le prononcent) que celui fait par ceux qui le lisent (ou l'entendent). Au lieu de leur « usage », Wittgenstein parle également de façon à peu près équivalente du « rôle » des signes dans le jeu, parfois également de leur « fonction ${ }^{37}$. On décrit le rôle d'un signe (le fait que ce soit par exemple le nom d'une personne) en disant que c'est le deuxième signe inscrit sur le bout de papier ; que, lorsque $\mathrm{B}$ entre dans le magasin, il le prononce à haute voix ; que l'un des employés vient alors vers lui, etc.

Prononcer un mot (un signe isolé) ne constitue pas encore un "mouvement" dans un jeu de langage. Celui-ci va consister à énoncer une proposition, c'est-à-dire un signe « complet ${ }^{38}$. On peut parler non seulement de la signification (c'est-à-dire de l'usage ou du rôle) des signes incomplets (des noms de personnes, de nombres, etc.) mais de celui des propositions dans

vie ? C'est dans son usage qu'il vit. »

36. $B B$, p. 172 (je souligne) ; $c f$. aussi $P U, \S 37$.

37. $C f . P U, \S 11,17,556$ et 559 .

38. $B B$, p. $82 ; c f$. aussi $B B$, p. $77-78, P U, \S 19$ et $W L C$, p. 11-12. 
un jeu de langage ${ }^{39}$. Un énoncé complet peut être formé d'un seul signe non analysable (ainsi « Poutre! » ou « Dalle !» dans le jeu du constructeur). Wittgenstein fait une distinction entre deux composantes de la signification d'un énoncé. La première est sa composante illocutoire. Dire « Poutre! " revient à donner un ordre en vertu du « rôle que [son] énonciation [...] joue dans le jeu de langage ${ }^{40} »$. Pour décrire cette première composante (pour indiquer en quoi consiste un ordre), on dit par exemple que $\mathrm{A}$ se trouve dans une situation où il n'y a plus de poutres, mais où il en aurait besoin pour poursuivre son travail ; que A est le seul des deux individus qui puisse faire des énoncés de ce type-là (parce que, supposons, un aide n'est pas habilité à donner des ordres); que $B$ réagit toujours de telle ou telle façon (il va chercher des poutres, des dalles, etc.) quand il entend A donner les ordres, etc. La seconde composante est le contenu ou « radical » propositionnel » (Satzradikal) $)^{41}$. Le contenu de «Poutre! " pourrait se traduire en français par l'expression " Aller chercher une poutre $»:$ on décrit cette seconde composante en disant que, lorsque $B$ entend " Poutre! !, il agit en allant chercher une poutre (plutôt qu'une dalle, une brique, etc.).

Un passage des Recherches décrit une «extension » du jeu du constructeur dans laquelle il y a d'autres types de mots et une forme rudimentaire de syntaxe ${ }^{42}$. Outre « dalle », "poutre ", etc., le constructeur emploie des noms de nombres (représentés par la série de lettres «a », «b», «c ", ...) et des signes se traduisant en français par « là » et « ici ». Un autre «instrument » dans le jeu est un tableau d'échantillons de couleurs ${ }^{43}$. A donne par exemple à $B$ un ordre qui se formule «D-dalle-là ! » en lui montrant un des échantillons et en lui indiquant un endroit à proximité. Pour dire quel est le rôle (donc la signification) de l'énoncé dans le jeu ou de son équivalent français « Apporte quatre dalles de cette couleur et dépose-les là ! », on décrit la façon dont B exécute l'ordre : il se rend là où sont entreposées les dalles, il en prend quatre de la couleur demandée (après, supposons, les avoir comptées à haute voix et comparées à l'échantillon) et vient les déposer à l'endroit indiqué. On décrit plus précisément le rôle des mots comme suit : quand $A$ en indiquant du doigt un endroit demande à $\mathrm{B}$ d'aller chercher un objet et de venir le déposer " là ", $B$ va chercher l'objet et le dépose à cet endroit ; quand il lui demande de rapporter « $d$ » objets d'un certain type, B va en chercher quatre ; lorsqu'il lui commande de rapporter des objets d'une certaine couleur, B va chercher les objets ayant cette couleur en utilisant le tableau d'échantillons, etc.

On spécifie le "rôle " d'un énoncé en se référant aux activités des locuteurs (cf. section 1) mais aussi, plus généralement, aux «occasions » ou «cir-

39. Cf $B B$, p. 82

40. $P U, \S 21$.

41. $P U, \S 22$, texte inséré en bas de page.

42. $C f . P U, \S 8$.

43. $C f$. aussi $P U, \S 16$. 
constances » entourant son énonciation : ce sont, dit Wittgenstein, «les circonstances dans lesquelles une expression est employée qui constituent [make] sa signification $[\ldots]^{44} "$. Supposons que nous veuillons traduire un mot employé dans une tribu dont nous ne connaissons pas la langue. Comment savoir si un certain mot français « $M$ » sera la traduction correcte ? «Un critère de ce que signifient [les membres de la tribu] serait les occasions dans lesquelles ils emploient le mot que nous sommes enclins a traduire par ["M"], le rôle que, pourrions-nous dire, joue le mot [...] dans la vie de la tribu ${ }^{45}$. »

Pour citer un demier exemple tiré du Cahier brun, des personnes dans une tribu font passer des « tests » à des recrues pour savoir si elles sont aptes à aller au combat. Les " examinateurs" font entre autres des énoncés qu'on traduirait par «Effectuez le test du lancer du boomerang! ». Comment savoir si cette traduction est correcte? On lit ceci :

[Les] ordres [des examinateurs] commencent tous par une expression [...] que je pourrais traduire par les mots français « Effectuez le test ». Et cette expression est suivie d'une autre expression employée durant les combats pour désigner certaines actions. Ainsi, il y a l'ordre que les hommes exécutent en lançant leur boomerang et que par conséquent je devrais traduire par «Lancez les boomerangs! ». Par ailleurs, si un homme décrit la bataille à son chef, il utilise à nouveau l'expression que j'ai traduite par « Lancez les boomerangs ! " cette fois dans une description. Maintenant, ce qui caractérise un ordre en tant que tel ou une description en tant que telle ou une question en tant que telle, etc., est [...] le rôle que [son] énonciation [...] joue dans la pratique entière du langage ${ }^{46}$.

Plus généralement :

[Le] fait qu'un mot du langage de notre tribu se traduise correctement par un certain mot français dépend du rôle que joue le mot dans l'ensemble de la vie de la tribu, des occasions dans lesquelles il est employé, des expressions d'émotions dont il est généralement accompagné, des idées qu'il éveille habituellement ou qui provoquent son énonciation, etc. ${ }^{47}$

L'énoncé des examinateurs se traduit par «Effectuez le test du lancer du boomerang! $»-\mathrm{il} \mathrm{a}$ cette signification - parce que (au sens où on mentionne un critère) ceux-ci emploient régulièrement l'expression qu'on traduirait par "Effectuez le test ... " dans une situation où ils font passer des tests à des recrues et que celle-ci est suivie d'une autre expression (le radical propositionnel) référant à des actions qu'on observe pendant les exercices ou les combats. D'autre part, il revient à donner un ordre (et non à poser une question, faire une description, etc.), parce que seul l'examinateur peut dans ce genre de situation donner des ordres (compte tenu des institutions de la tribu) et que les hommes agissent (à peu près) toujours immédiatement dès qu'ils l'ont entendu en effectuant les actions décrites par le radical propositionnel.

44. $B B$, p. 104.
45. $B B$, p. 94 (je souligne).
46. $B B$, p. $102-103$ (je souligne).
47. $\quad B B$, p. $103 ; c f$. aussi $W L C$, p. $102-103$. 
Les « circonstances » sont par exemple des situations où on fait passer des tests, des actions (lancer un boomerang), des « expressions d'émotions », des « idées » et même « les gestes, les jeux de physionomie et [...] le ton de voix [des locuteurs] ${ }^{48} \gg$.

En somme, l'énoncé du constructeur se traduit par «Va chercher une poutre ! " (il a le même contenu que "Va chercher une poutre ! " en français) en raison de son "rôle" dans le jeu de langage (être le nom d'une maison dans le jeu de langage du Cahier brun cité plus haut a pour critère et n'est rien d'autre que le fait d'avoir été peint sur la porte d'une maison, le fait que le signe soit inscrit sur une des listes que A remet à B, que B se dirige vers la maison quand il voit le signe, etc.). Un jeu de langage comprend normalement l'énonciation de mots, les activités des locuteurs (telles qu'exécuter des ordres) et les autres «traits de la situation " (features of the situation) ${ }^{49}$ qui fixent ensemble la signification (la force illocutoire et le contenu propositionnel) des énoncés, entre autres la position (au sens social ou institutionnel) des participants (du constructeur vis-à-vis de son aide, des examinateurs vis-à-vis des recrues), leurs « expressions d'émotions », leurs « idées », etc. Bref, un signe n'est une expression (un symbole) que s'il est employé dans un jeu de langage ${ }^{50}$. C'est ce que veut dire Wittgenstein quand il affirme que la signification est l'usage ${ }^{51}$.

\section{Jeux de langage et langues naturelles}

Quelle relation y a-t-il entre jeux de langage et langues naturelles? Pour Wittgenstein, (a) une langue naturelle est un système de communication beaucoup plus « complexe " ou " développé » que, par exemple, le langage des constructeurs et $(b)$ une langue naturelle est une collection de jeux de langage. Je citerai des textes à l'appui de $(a)$ et de $(b)$.

(a) On lit dans le Cahier bleu: « [N]ous reconnaissons [dans les jeux de langage] des formes de langage qui ne sont pas séparées par une coupure de

48. $B B$, p. 103.

49. $B B$, p. 104 .

50. $C f$. aussi $P U, \S 261$.

51. Pour revenir à l'interprétation de Hunter, on pourrait m'objecter en faveur de son interprétation que Wittgenstein fait la remarque que c'est dans des contextes où l'on donne des explications de la signification que figure le concept de signification. On lit dans les Recherches: “"La signification d'un mot est ce qu'explique [sa] signification". C'est-à-dire : si vous voulez comprendre l'usage du mot "signification", regardez ce que l'on appelle "explication de la signification". " $(P U, \S 560 ; c f$. aussi p. $B B, 1)$ Mais sa remarque n'est pas incompatible avec ma lecture du slogan "La signification, c'est l'usage ». Dans la Grammaire philosophique, on lit : « La signification d'un mot est ce que l'explication de la signification explique. [...] L'explication de la signification explique l'usage du mot. L'usage du mot dans le langage est sa signification. " $(P G$, p. 59-60) On trouve ici les deux affirmations : la signification est ce qu'explique l'explication de la signification et la signification est l'usage. 
nos [formes] plus complexes. Nous voyons que nous pouvons construire [build up] les formes compliquées à partir des [formes] primitives en [leur] ajoutant graduellement des formes nouvelles ${ }^{52}$." De même, on lit dans la Grammaire philosophique : il n'y a pas « de frontière nette entre les formes primitives et [les formes] plus complexes $[\ldots]^{53} \gg$. On peut trouver un certain nombre d'exemples de «constructions » dans la première partie du Cahier brun $^{54}$; dans les Recherches, un exemple est celui déjà cité du jeu des constructeurs et de son « extension » dans le paragraphe $8^{55}$.

(b) Les Recherches proposent l'image suivante d'une langue naturelle : "Notre langage peut être regardé comme une cité ancienne : un dédale de petites rues et de places, d'anciennes et de nouvelles maisons, et de maisons avec des ajouts d'époques différentes, entourées d'une multitude de nouvelles banlieues aux rues droites et régulières et aux maisons uniformes ${ }^{56} \%$. On trouve une image semblable dans un passage du Cahier brun: "Nous nous représentons le langage d'un adulte comme une masse nébuleuse de langage, sa langue maternelle, entourée de jeux de langage distincts [discrete] et

52. $B B$, p. 17

53. $P G$, p. 62 .

54. Cf. également $W L C$, p. 101-105.

55. Dans sa préface aux Cahiers bleu et brun, Rush Rhees cite un passage d'un manuscrit non publié dans lequel Wittgenstein semble dire quelque chose de très différent ; le passage se lit en partie comme suit : "Lorsque je décris certains jeux de langage simples, ce n'est pas dans le but de construire [aufzubauen] graduellement à partir de ceux-ci les processus du langage développé $[\ldots]$, ce qui ne peut que mener à des injustices [Ungerechtigkeiten] (Nicod et Russell) [...]. " (BB, préface, p. viii). L'allusion à Nicod et Russell signifie apparemment que l'on n'a pas affaire ici à une construction au sens d'une « reconstruction», du genre de celles qu'ont envisagées certains philosophes, du langage ordinaire ou peut-être du langage de la science à partir de formes de langage plus simples. En quel autre sens, dans ce cas, parler d'une "construction »? Après avoir cité des exemples d' « extensions» d'un jeu de langage simple, Wittgenstein fait remarquer dans l'un de ses cours : « Ne commettez pas l'erreur de supposer que je montre ici comment le langage est construit [built up] ou de la façon dont il a évolué. " Et il ajoute : «Parfois, il est plus facile d'imaginer ces langages inventés comme des langages d'une tribu primitive et parfois comme le vrai [actual] langage primitif d'un enfant. Un enfant commence réellement par un tel langage primitif. Son apprentissage du langage prend surtout la forme de tels jeux. Un nouveau jeu introduit un nouvel élément dans le langage [...].» (WLC, p. 105) Wittgenstein veut dire apparemment que les exemples d' « extension » à partir de formes de langage simples sont, bien entendu, des exemples fictifs, mais les premières formes de langage inculquées aux enfants sont $d u$ même type que ces jeux de langages et leur apprentissage suit un processus en gros semblable à celui décrit dans le $\mathrm{Ca}$ hier brun. De façon similaire, les langues naturelles se sont développées à partir de systèmes de communication semblables à celui du jeu du constructeur par adjonction graduelle de formes nouvelles mais on ne doit pas supposer que les exemples de « constructions » du Cahier brun reflètent la façon dont les langues naturelles ont effectivement évolué à partir de ces formes primitives.

56. $P U, \S 18$. 
plus ou moins bien délimités [clear-cut], les langages techniques ${ }^{\text {T }}$. " Des exemples des «banlieues de notre langage " (de langages techniques) seraient « le symbolisme de la chimie » et « la notation du calcul infinitésimal ${ }^{58}$ ". En quoi consiste la partie « centrale » d'une langue si les langages techniques en forment la " périphérie " ? D'après le texte des Recherches, elle consiste en des jeux tels que « donner des ordres », " rapporter des événements » (durant une bataille), «poser des questions et y répondre ${ }^{59}$ » ou « faire des affirmations ${ }^{60} »$. Dans un cours qui reprend la même image, Wittgenstein dit: "Entourant l'ensemble flou du langage ordinaire, il y a les langages spéciaux, par exemple les langages de la chimie et de la météréologie. » Il ajoute : « Je considérerai un langage comme un tel agglomérat [conglomerate]. Dans notre langage, nous trouvons un mélange [mixture] de descriptions, d'hypothèses, de questions, d'ordres, etc. $[. . .]^{61}$ ». Ainsi, l'« en semble flou » (le « dédale », la «nébuleuse ») d'une langue naturelle est formée d'une quantité « innombrable $"^{62}$ de jeux de langage ; de plus, «n'importe quelle liste que nous pourrions en donner serait totalement inadéquate ${ }^{63}$ ".

Pourquoi sont-ils « innombrables "? Les exemples du paragraphe 23 des Recherches sont instructifs. On trouve non seulement les jeux « donner des ordres et y obéir ", " relater un événement », " faire des conjectures sur cet événement ", " décrire l'apparence d'un objet ", " demander quelque chose ", (ou « remercier», « jurer», ou « prier»), mais des jeux tels que « construire un objet d'après une description (ou un dessin) ", " émettre une hypothèse et la tester " ou « jouer une pièce de théâtre ». On pourrait appeler les activités du premier groupe des "actes de langage" et celles du second groupe des "activités linguistiques impures" (j'emprunte l'expression à George Pitcher) ${ }^{64}$ dans la mesure où elles comportent beaucoup d'autres choses (en particulier des actions, des comportements) que la seule action de prononcer ou d'inscrire des signes (il y a dans l'acte d'émettre une hypothèse et de la tester une partie «non linguistique " plus importante que dans celui de faire une promesse ou de décrire quelque chose - bien que, comme le dit Pitcher, il n'y ait pas une distinction nette entre les deux types d'activités). Mais si on peut appeler « jeux de langage » les activités d' « émettre une hypothèse et la tester " et de « jouer une pièce de thêâtre ", il semble qu'on puisse en faire autant de pratiques telles que «se marier» (et " divorcer »), " encaisser un

57. $B B$, p. 81 .

58. $P U, \S 18$.

59. $C f . P U, \S 19$.

60. $C f . P U, \S 21$

61. $W L C$, p. $102 ; c f$. aussi $B B$, p. 67-68.

62. $P U, \S 23$.

63. WLC, p. 102

64. G. Pitcher, The Philosophy of Wittgenstein, Englewood Cliffs, Prentice-Hall, 1964, p. 239-240. Cf. également P. M. S. Hacker et G. P. Baker, Wittgenstein: Understanding and Meaning, Oxford, B. Blackwell, 1980, p. 97-98. 
chèque dans une banque ", " donner un cours ", " contracter une assurance ", "postuler un emploi » ou " voter (lors d'une élection) » - qui sont toutes des activités dans lesquelles on fait usage de signes en apposant une signature, en échangeant des paroles, en inscrivant un « $\mathrm{x}$ » sur un bulletin de vote, etc. C'est apparemment l'une des raisons pour lesquelles Wittgenstein pense qu'il n'est pas possible de dresser la liste exhaustive de tous les jeux de langage constituant une langue naturelle.

Bref, on peut décrire comme suit la structure d'une langue : c'est une collection (un " agglomérat») de jeux de langage. Tous les usages extrêmement variés d'une langue se décrivent à l'aide de verbes tels que « faire une promesse ", « émettre une opinion ", « donner des ordres et y obéir », « poser une question » ou « proposer des devinettes » et de verbes désignant des pratiques telles que "se marier ", " encaisser un chèque », etc. On peut aussi imaginer pour certains jeux des formes plus simples que les formes familières: le jeu des constructeurs représente une variante (qui pourrait être qualifiée de plus " simple » ou plus " primitive ») du jeu " donner des ordres et les exécuter » joué par les locuteurs du français.

\section{La signification dans les langues naturelles}

D'après ce qui précède, Wittgenstein soutient, d'une part, que la signification (le contenu sémantique) d'une expression est son rôle dans des jeux de langage (section 2) et, de l'autre, qu'une langue naturelle est une collection de jeux de langage (section 3). Il semble qu'on puisse en déduire que la signification d'une expression dans une langue naturelle est son usage dans cette langue (son rôle dans les jeux de langage qu'on joue en la parlant) au même sens où il est dit que la signification de "Poutre!" est son usage dans le jeu $d u$ constructeur. Je développerai à présent cette thèse centrale mais largement implicite des Recherches en examinant quelques uns des problèmes qu'elle soulève.

(1) La syntaxe d'une langue. La structure syntaxique des énoncés dans une langue naturelle détermine au moins en partie son contenu. Est-ce que la théorie de la signification-usage suffit à rendre compte de cette composante de la signification? J'ai fait allusion plus haut à ce problème (section 2). On peut poser entre autres deux sous-questions : (a) peut-on expliquer la diversité de fonction des mots (des «parties du discours », comme dit Wittgenstein) ${ }^{65}$, c'est-à-dire les différentes catégories syntaxiques de la langue, et $(b)$ en quel sens le contenu d'un énoncé est-il fonction de sa structure?

(a) L'exemple du jeu de langage du paragraphe 8 des Recherches suggère une réponse. Pourquoi l'ordre du constructeur «D-dalle-là ! » se traduit-il par (signifie-t-il la même chose que) "Apporte quatre dalles et dépose-les là !»? Il a cette signification parce que (au sens où on cite un critère), pour 
exécuter l'ordre, $\mathrm{B}$ se rend là où sont entreposées les dalles, il en prend quatre après avoir énuméré les lettres «a, b, c, d », et va les déposer à l'endroit indiqué. D'autre part, étant donné le rôle général des signes (leur type d'usage) dans le jeu, « $d$ » doit être interprété comme un nom de nombre (on aurait pu lui substituer les signes « $a », 《 b »$, , $c$ »,..) et « dalle » comme un nom d'objet employé comme matériau de construction (il aurait pu être remplacé par " brique ", " poutre », etc.). On lit dans le Cahier brun : " [n]ous pouvons voir facilement une similitude dans l'usage des mots "un", "deux", "trois", etc. et également une similitude dans l'usage de "dalle", "colonne", "brique", etc., et c'est ainsi que nous distinguons entre les parties du discours ${ }^{66}$ ".

(b) Une propriété formelle d'un énoncé est constituée entre autres par l'ordre des mots. Une variante du jeu du constructeur serait celui dans lequel on a des phrases de la forme «Dalle, brique, poutre ! ${ }^{67}$. Lorsque A donne l'ordre à $\mathrm{B}, \mathrm{B}$ obéit en allant chercher d'abord une dalle, ensuite une brique et puis une poutre (je suppose que $B$ ne peut pas rapporter ensemble les trois objets). Ainsi la façon dont l'ordre est exécuté varie suivant l'ordre d'occurrence des mots dans la phrase. Comment expliquer la différence de contenu entre «Dalle, brique, poutre ! " et (disons) “ Poutre, brique, dalle ! " ? On peut supposer que le rôle des deux énoncés est fonction d'une part (et trivialement) de leur formulation (de la nature et de l'ordre de leurs constituants) et, de l'autre, des circonstances de leur énonciation - de leur rôle ou de leur usage. Puisque «Dalle, brique, poutre !» et «Poutre, brique, dalle ! n' ont pas le même rôle (les activités des locuteurs diffêrent), les énoncés n'auront pas la même signification. Mais comment savoir si la traduction correcte de « Dalle, brique, poutre ! " est «Va d'abord chercher une dalle, et puis une brique, et ensuite une poutre ! " et non à l'inverse « Va d'abord chercher une poutre, et puis une brique, et ensuite une dalle ! ? B peut se tromper en l'exécutant ou bien il est possible que l'ordre d'occurrence des mots soit indifférent et que B l'ait exécuté par hasard en effectuant les actions dans cet ordre. Wittgenstein pourrait répondre qu'un traducteur doit tenir compte d'autres occasions d'usage. Il doit faire plusieurs observations et formuler une hypothèse sur la traduction de l'énoncé compatible avec tous les usages observés. Si tel est le cas, il semble que la signification de l'énoncé soit fonction non seulement de ses circonstances particulières d'usage mais de la signification d'autres énoncés dans le jeu et, par conséquent, des circonstances dans lesquelles ont été faits ces autres énoncés.

(2) Le problème de la multiplicité des significations. Si le contenu sémantique est fonction du rôle dans un jeu de langage et si la plupart des mots d'une langue naturelle sont employés dans un très grand nombre de jeux, ceux-ci n'ont-ils pas autant de significations qu'il y a de jeux dans lesquels on les emploie? Wittgenstein fait allusion, semble-t-il, à ce problème à un en-

66. $B B$, p. $83 ; c f$. aussi $P U, \S 17$.

67. $C f . B B$, p. 83. 
droit de la Grammaire philosophique où il écrit : " [O]n pourrait dire de l'usage du mot "bon" (au sens éthique) qu'il est composé d'un très grand nombre de jeux apparentés [verwandter] les uns aux autres; pour ainsi dire les facettes de [son] usage. Mais c'est précisément le lien entre ces facettes, leur parenté [Verwandtschaft], qui donne lieu ici à un concept ${ }^{68}$. "Il existe, autrement dit, une multitude d'usages du mot « bon » (un très grand nombre de jeux dans lesquels on l'emploie), mais ceux-ci sont reliés par des relations de « ressemblance de famille». Pour prendre un autre exemple, on ne trouve rien qu'ont en commun tous les usages de « rouge » bien que plusieurs de ces usages aient ceci de commun que les énoncés dans lesquels figure « rouge " sont faits en présence d'objets rouges.

(3) La signification des phrases déclaratives. Supposons qu'un énoncé dans une langue étrangère signifie «Ce ballon est rouge ". Comment savoir si "Ce ballon est rouge » en français est la traduction correcte - comment savoir quel est le rôle de l'énoncé ou quelles en sont les circonstances d'usage ? D'autres traductions pourraient être « Le ballon est sphérique ", «L'arbre (au pied duquel se trouve le ballon) est haut ", etc. Car il faut compter au nombre de ces circonstances les états de choses que le ballon est sphérique ou que l'arbre est haut, et non seulement que le ballon est rouge. Wittgenstein, je pense, pourrait répondre comme suit.

La traduction d'une langue (radicalement) étrangère suppose qu'on ait d'abord compris le sens des activités des locuteurs, comme on le lit dans ce passage des Recherches:

[...] Supposez que vous veniez comme explorateur dans une contrée inconnue dont la langue vous est complètement étrangère. Dans quelles circonstances diriez-vous que les gens qui vivent là donnent des ordres, les comprennent, refusent d'y obéir, etc. ?

La façon d'agir commune des êtres humains est le système de référence au moyen duquel nous interprétons une langue étrangère ${ }^{69}$.

Wittgenstein explique ensuite cela de la façon suivante :

Imaginons que les gens dans ce pays mènent les activités humaines courantes et ce faisant se servent apparemment d'un langage articulé. Quand nous observons leur comportement, nous le trouvons intelligible, il nous paraît « logique ». Mais si nous essayons d'apprendre leur langue, nous constatons que ce n'est pas possible. Car il n'y a pas chez eux de connexion régulière [regelmässiger Zusammenhang] entre les paroles, les sons, et les actions. [...]

Devrions-nous dire que ces gens possèdent un langage ; qu'ils donnent des ordres, communiquent des informations, et ainsi de suite ?

À ce que nous appelons « langage», il manque la régularité [die Regelmässigkeit] ${ }^{3(t}$.

68. $P G$, p. 77.

69. $P U, \S 206$.

70. $P U, \S 207$. 
Si on observe le constructeur et son aide, on peut comprendre leurs activités (on sait qu'ils construisent un abri, que l'un apporte les objets pour que l'autre les utilise, etc.). On peut en inférer certaines croyances, intentions ou désirs. Si A est à court de briques (il n'y a plus de briques, il a interrompu son travail, etc.), on peut supposer qu'il va former le désir que B lui en apporte et, s'il fait l'énoncé "Va chercher des briques ! et celui-ci est suivi de l'action appropriée de B, on aura des raisons de penser que l'énoncé est un ordre et que son contenu est le même que celui de la phrase «Va chercher des briques! ». Bref, si on comprend les actions des locuteurs et on peut voir le lien (le regelmässiger Zusammenhang) entre les sons qu'ils émettent et leurs actions, on peut aussi interpréter leur langage (ce qui, bien sûr, n'est qu'une autre façon de dire que la signification des énoncés est leur usage). La question est de savoir si cela est vrai également des énoncés déclaratifs.

Supposons que nous observions les actions des membres d'une tribu qui vont chasser. La veille, ils ont préparé leurs armes, ils ont fait des exercices de lancer du javelot, de tir à l'arc, etc. Le lendemain, pendant la chasse, ils font des assertions telles que « Ce sont les traces d'un sanglier» ou «L'animal n'est plus très loin ». Nous pouvons dans bien des cas comprendre leurs actions - elles sont «intelligibles». Si un chasseur se penche et examine des traces sur le sol, nous comprenons son comportement ; de plus, nous supposons que cela va produire chez lui de nouvelles croyances qu'il va parfois exprimer verbalement (si par exemple un autre chasseur le fixe du regard avec un air interrogatif). Nous pourrons ainsi donner une traduction au moins approximative de plusieurs de leurs énoncés. Un énoncé va se traduire par la phrase "Ce sont les traces d'un sanglier ", un autre par "L'animal n'est plus très loin " (les chasseurs ont entendu des bruits provenant d'un endroit où se trouve apparemment l'animal), etc.

Le problème est que cela ne tient pas pour tous les énoncés déclaratifs. Pour reprendre un exemple du Cahier Brun (cf. section 2), un guerrier relate devant son chef les péripéties d'une bataille et dit entre autres « C'est alors que les guerriers ont lancé leurs boomerangs » : il est clair dans ce cas que les circonstances ne permettent pas de connaître la signification de l'énoncé. Mais supposons qu'il y ait un autre jeu dans lequel ils donnent des ordres (je suppose pour simplifier que les contenus propositionnels sont inanalysables : un signe propositionnel signifie "Lancer les boomerangs", un autre "Se préparer au combat», etc.). Si un traducteur a réussi à interpréter des ordres tels que "Lancez les boomerangs! ", "Préparez-vous au combat!", etc., il sera probablement aussi en mesure de traduire des assertions rapportant des événements passés. Or si la traduction d'énoncés faits dans un jeu suppose qu'on ait au préalable traduit des énoncés faits dans un autre (si on comprend "Ils ont lancé leurs boomerangs" seulement si on a compris "Lancez les boomerangs !»), alors le contenu (le rôle) des énoncés faits dans un jeu (celui où le guerrier fait des descriptions) dépend du contenu des énoncés correspondants (ayant le même radical propositionnel) faits dans d'autres jeux (ceux 
par exemple où ils donnent des ordres). C'est peut-être cela, au moins en partie, que suggère Wittgenstein lorsqu'il écrit dans le Cahier brun, dans un passage déjà cité : « le fait qu'un mot du langage de notre tribu se traduise correctement par un certain mot français dépend du rôle que joue le mot dans l'ensemble de la vie de la tribu $[\ldots]^{71}$ ".

Une conséquence qui s'ensuit est que les jeux de langage qui forment une langue naturelle ne sont pas « complets » comme l'est le jeu du constructeur (cf. section 1). Il est "complet " en ce sens que les énoncés de A ont une signification (ils ont un rôle dans le jeu) bien que les seuls mots employés soient " poutre ", «brique » ou " dalle » et qu'ils ne servent qu'à donner des ordres (il est, par conséquent, indépendant des autres jeux). Mais un traducteur comme dans l'exemple précédent ne peut comprendre "Ils ont lancé leurs boomerangs » s'il n'a pu d'abord traduire l'ordre «Lancez les boomerangs !». Il semble par conséquent qu'un grand nombre de jeux dans une langue naturelle ne soient pas complets (et donc indépendants d'autres jeux) au sens où est supposé l'être le jeu des constructeurs.

(4) L'apprentissage d'une langue. Je mentionnerai deux problèmes. (a) Apprendre une langue, c'est apprendre à jouer des jeux de langage. Mais une langue naturelle est constituée d'une quantité « innombrable » de jeux de langage (le jeu dans lequel un constructeur donne des ordres à son aide, celui dans lequel le chef d'une tribu donne des ordres aux guerriers, celui dans lequel les examinateurs donnent des ordres aux recrues, etc.) Comment apprendre une langue est-il possible ? On pourrait répondre que si connaître une langue suppose la maitrise d'un très grand nombre de jeux de langage, cela ne veut pas dire que chaque jeu doive être appris isolément. Quelqu'un pourrait avoir appris le jeu du constructeur (dans lequel A donne des ordres à B), mais non le jeu dans lequel un locuteur promet de (ou s'engage à) aller chercher une dalle ou une poutre. S'il peut jouer le jeu dans lequel les locuteurs font des promesses (dans une situation différente), il n'aura pas à apprendre à jouer le jeu « promettre d'aller chercher des dalles, des poutres, etc. » : il peut être suffisant qu'il ait déjà maîtrisé ces autres jeux.

(b) Au cours de l'apprentissage d'une langue, dit Wittgenstein, on apprend non seulement les jeux «donner des ordres », " faire des promesses », ou « décrire des objets », mais des jeux tels que « formuler une hypothèse et la tester ", "donner un cours », ou " voter lors d'une élection ». Mais, normalement, un enfant ne sait pas jouer le jeu «formuler une hypothèse et la tester » ou « donner un cours » et tous les adultes ne connaissent pas le symbolisme de la chimie ou du calcul infinitésimal. Est-ce que cela veut dire qu'ils ne possèdent pas la maîtrise de leur langue ? Wittgenstein pourrait faire appel à la distinction entre la partie «centrale » d'une langue et sa "périphérie » (on pourrait dire d'un enfant de six ans qu'il maîtrise le français parce qu'il sait jouer les jeux appartenant à la partie centrale de sa langue). Mais la distinction

71. $B B$, p. 103 , (je souligne). 
n'est pas évidente (il n'y a pas une différence stricte entre les activités linguistiques " pures » et « impures » ou entre les jeux de langage techniques et les jeux non techniques). Une autre réponse consisterait à dire qu'il n'y a rien de tel à proprement parler que la maîtrise d'une langue. Un enfant de six ans a une meilleure connaissance de sa langue maternelle qu'un enfant de trois ans mais il connaît moins de jeux de langage que la plupart des adultes. Il s'ensuit qu'aucun locuteur ne maîtrise parfaitement sa langue maternelle (personne ne connaît tous les jeux joués par tous les locuteurs de sa langue).

On pourrait objecter que la difficulté découle d'une conception erronée de la structure d'une langue et soutenir qu'une langue se définit non comme un « agglomérat » de jeux de langage mais, plutôt, comme un ensemble d'éléments lexicaux et de règles de formation et d'interprétation des phrases. Quand quelqu' un apprend à jouer le jeu « formuler une hypothèse et la tester » ou « donner un cours » (ou « se marier », « voter lors d'une élection», etc.), il n'acquiert pas ce faisant une meilleure connaissance de sa langue, mais de nouvelles façons d'agir ou de se comporter (comme on apprend à scier une planche ou à faire de la voile) : l'apprentissage du jeu « formuler une hypothèse et la tester " présuppose une connaissance de la langue (pour qu'on puisse formuler des hypotheses) mais il n'ajoute pas à cette connaissance. La réponse de Wittgenstein, je pense, serait qu' une langue est bien une collection de jeux de langage dans la mesure où un signe dans une langue a une signification (c'est un authentique symbole) à la condition seulement qu'il ait un usage - et posséder un usage, c'est jouer un rôle dans un jeu de langage.

\section{Quelques objections}

Je répondrai pour terminer à des objections qu'on pourrait opposer à mon interprétation.

(1) J'ai cité au début un passage des Recherches dans lequel Wittgenstein nie apparemment qu'une théorie du langage est possible. Mais il me semble qu'il conteste plutôt à cet endroit la possibilité d'une explication scientifique (empirique et causale) de l'essence du langage. Des interprètes des Recherches ont fait une autre objection apparentée. Warren Goldfarb écrit : « [J]e ne lis pas [la] célèbre "définition" de la signification en tant qu'usage du paragraphe 43 comme une définition ou une explication [de l'idée de signification] ou comme proposant une "théorie de la signification basée sur le concept d'usage"72 . " " Je soutiendrais, dit Goldfarb, qu'il n'y a pas de notion générale d'usage et je serais prêt à affirmer que Wittgenstein est d'accord ${ }^{73}$." Puisqu' « invoquer [le concept] d'usage dit en soi peu de choses [carries little information], la remarque du paragraphe 43 revient essentiellement à nier la

72. W. Goldfarb, «I Want You to Bring Me a Slab : Remarks on the Opening Sections of the Philosophical Investigations », p. 279.

73. Ibid., p. 279. 
possibilité et la pertinence [de théories] de la signification ${ }^{74} »$. James Bogen fait une remarque similaire ; il écrit : " [...] je crois qu'il est incorrect de dire que Wittgenstein se proposait de donner une theorie positive globale [a single positive theory] de l'usage du langage $\mathrm{e}^{75}:$ » " [n]otre emploi du langage est trop diversifié [selon lui] pour qu'une théorie générale soit possible [...] » ${ }^{76}$.

Il semble que le concept d'usage soit trop " général " pour servir de base à une théorie du langage. Mais l'interprétation de Goldfarb, à mon avis, est erronée. Le concept important n'est pas tant le concept d'usage que celui du rôle dans un jeu de langage. Wittgenstein invoque la notion d'usage pour mettre en évidence les activités des locuteurs comme constitutives de la signification des signes. Quand il dit que la signification est l' « usage», il n'emploie pas le mot dans son acception habituelle (qui est effectivement très générale : on peut parler de l' "usage » d'un outil, d'un véhicule, d'un ingrédient, d'un matériau, d'un meuble, d'un équipement, etc.) mais dans un sens quasi-technique (ce qui est plus clair si on pense à l'expression " rôle dans un jeu de langage »). "Usage » est aussi employé dans les Recherches en un sens général $^{n}$, mais ce n'est pas en ce sens qu'il affirme que la signification est l'usage. D'autre part, je ne trouve pas plausible l'interprétation de Goldfarb lorsqu'il prétend que la remarque du paragraphe 43 revient à nier « la possibilité et la pertinence " d'une théorie du langage : il me semble que c'est un peu comme si, pour Goldfarb, Wittgenstein disait : «J'affirme que la signification est l'usage bien que cela ne veuille pas dire grand-chose étant donné la généralité (la vacuité, veut dire apparemment Goldfarb) du concept d'usage ". C'est par ailleurs avec raison, je pense, que Bogen fait remarquer qu'il y a pour Wittgenstein une « diversité » considérable d'usages des mots dans une langue ; mais il ne s'ensuit pas l'impossibilité d'une théorie du langage : en soutenant que la signification est l'usage, Wittgenstein ne veut évidemment pas dire que tous les énoncés (les expressions) d'une langue ont le même usage.

(2) Pour Rhees, le jeu du constructeur n'est pas ce qu'on appellerait au sens ordinaire un langage ( $c f$. section 1). Bogen écrit : "Comme le fait remarquer Rhees, les descriptions de jeux [de langage] de Wittgenstein leur donnent un air suspect [d'activités] mécaniques et stéréotypées, remarquablement dépourvues du genre de surprises et d'improvisations qui caractérisent la plupart de nos activités pratiques et du langage qu'elles impliquent ${ }^{78}$. $»$ Mais, si tel est le cas, il est faux de dire que les langues naturelles sont des collections de jeux de langage. Pour Bogen, « [d]écrire l'emploi de mots du langage ordinaire en termes de rôles dans des jeux de langage présuppose que [celui-

74. Ibid., p. 279

75. J. Bogen, Wittgenstein's Philosophy of Language, p. 213.

76. Ibid., p. 214.

77. Cf. par exemple $P U, \S 44$.

78. Ibid., p. 201. 
ci] soit une collection d'activités isolées [discrete] et stéréotypées au même titre [que le jeu du constructeur] et que ces activités épuisent notre emploi des mots [dans le langage courant] ${ }^{79} »$. (Bogen ne précise ce qu'il entend par l'expression «rôles dans des jeux de langage », mais je supposerai qu'il l'interprète comme je l'ai suggéré plus haut.) Bref, une langue naturelle, pour Wittgenstein, n'est pas une collection de jeux de langage parce que ceux-ci sont des formes d'activités « isolées », " mécaniques » et « stéréotypées ». Je ferai deux remarques.

(a) L'impression que partagent apparemment Rhees, Black et Bogen que les jeux de langage «simples » ont peu de choses à voir avec nos formes ordinaires de langage s'explique, je pense, en partie par une description erronée du jeu des constructeurs. Rhees le décrirait probablement comme suit : A émet un son ou un signe faisant partie d'un répertoire de signes ; B réagit en exécutant une action, et il agit (à peu près) toujours de la même manière quand il entend un des sons. Si cette description était exacte, les signes seraient peut-être semblables, comme le dit Rhees, à des " signaux » (ou peut-être au langage de certaines espèces animales). Mais je pense qu' une meilleure description serait celle-ci : (i) A construit une habitation (ou un abri, etc.) avec l'assistance d'un aide ; (ii) ils ont des désirs, des intentions et des croyances (A veut construire une habitation, il désire que B aille chercher des dalles, etc.) ; (iii) lorsque A dit «Dalle! », son énoncé revient à donner un ordre et, en allant chercher une dalle, B obéit à l'ordre. Il est clair d'après ce qui précède que la seconde description est la description correcte (cf. sections 2 et 4 ).

(b) En quel sens qualifier de «mécaniques » ou de «stéréotypées » les activités des constructeurs ? Au sens apparemment où chaque fois que $A$ émet un signe pour donner un ordre, B effectue l'action appropriée et aucune autre action dans le jeu n'est possible. La difficulté, semble-t-il, est que les choses ne se passent pas toujours de cette façon-là dans nos échanges linguistiques courants. Mais je ne vois pas pourquoi cela poserait un problème pour Wittgenstein. Premièrement, lorsque quelqu'un donne un ordre (si les conditions pour qu'un ordre soit donné sont remplies), celui-ci est normalement exécuté (si quelqu'un promet de faire quelque chose, il va dans les cas normaux respecter sa promesse) : les descriptions de jeux de langage font abstraction des malentendus, oublis, accidents, etc.. Deuxièmement, le jeu des constructeurs peut sembler «mécanique » ou «stéréotypé » dans la mesure où il est " simple", étant donné entre autres le peu de variation du contenu des énoncés ("Apporte une brique! », " Apporte une dalle ! », etc.). Je pense que Wittgenstein pourrait faire remarquer que nos jeux de langage sont effectivement des formes de langage plus " complexes" ou " développés ", c'est-àdire beaucoup plus riches, que le jeu du constructeur, mais que cela n'entraine aucune différence importante entre ces formes de langage (il soutiendrait que la signification des énoncés dans les deux cas est leur usage) ( $c f$. section 3).

79. Ibid., p. 201. 
Troisièmement, on pourrait imaginer la situation suivante : en s'apprêtant à exécuter l'ordre d'aller chercher des briques, $B$ se rend compte qu'il n'y en a plus ; il revient vers A et lui dit «Il n'y a plus de briques. » Supposons que B n'a jamais fait auparavant une assertion ayant ce contenu ou fait des assertions pendant qu'il assiste $\mathrm{A}$ bien qu'il ait appris à faire des assertions dans d'autres genres de circonstances plus ou moins similaires ; en ce sens, il a dû « improviser » ( $c f$. section 4). Est-ce que cela pose un problème pour Wittgenstein ? Il ne suggère nulle part à ma connaissance que les locuteurs ne sont pas capables d' « improviser » (en faisant par exemple des assertions inédites) quand ils jouent des jeux de langage (et il pourrait à nouveau ici faire remarquer que cela n'est pas incompatible avec le slogan « la signification, c'est l'usage »).

(3) On pourrait faire à Wittgenstein l'objection suivante (en supposant cette fois correcte mon interprétation). Si le contenu d'un énoncé est son usage, il semble qu' on puisse en dire autant du " contenu " d'autres « instruments » utilisés dans des jeux de langage. On pourrait par exemple soutenir qu'un certain type d'objet est un échantillon de couleur rouge étant donné son usage (celui qu'en font par exemple A et B dans le jeu du paragraphe 8 des Recherches). Mais s'il a un usage, est-ce qu'il a aussi une signification? On lit également dans les Recherches à propos du jeu d'échecs : «Disons ceci : la signification [Bedeutung] d'une pièce est son rôle dans le jeu ${ }^{80}$. » Et, dans la Grammaire philosophique, il fait la remarque que ce sont les circonstances (y compris les règles) du jeu d'échecs qui donnent sa "signification" (Bedeutung) à une certaine configuration de pièces (par opposition à un arrangement quelconque de pièces de bois sur un échiquier) ${ }^{81}$. Mais on ne parle pas habituellement de la «signification " d'une piece (ou d'un mouvement) aux échecs, d'un échantillon de couleur ou d'une pièce de monnaie ${ }^{82}$.

Il n'est pas évident que Wittgenstein veuille dire d'une pièce aux échecs qu'elle a une signification au même sens qu'une expression dans une langue ; mais peut-il affirmer le contraire ? Il nie, d'une part, qu'il y ait quelque chose de commun à tous les usages du langage (quelque chose en quoi consisterait l'essence de tous les jeux de langage) : un énoncé n'a pas toujours une structure syntaxique ou un contenu propositionnel (une salutation, par exemple, n'en a pas); il ne sert pas toujours à l'expression de pensées ${ }^{83}$, etc. D'autre part, tous les signes ont une signification, laquelle est fonction de leur rôle dans des jeux de langage. Mais cela semble vrai également d'une pièce (ou d'une mouvement) aux échecs (d'un échantillon de couleur, d'une pièce de monnaie, d'une fleche peinte sur un panneau indicateur, etc.) : le roi aux échecs a ce «contenu» (le fait d'être le roi) étant donné son rôle dans le jeu (la flèche indique une direction en raison de l'« application » qu'en font les

\footnotetext{
80. $P U, \S 563$.

81. Cf. $P G$, p. $107 ;$; $f$. aussi $Z, \S 143$

82. Cf. $P G$, p. 107.

83. Cf. $P U, \S 304$.
} 
automobilistes, quelque chose est un échantillon de couleur en raison de son "rôle » dans les activités de ses utilisateurs, etc.). S'il n'y a entre divers jeux de langage que des « ressemblances de famille " et si une pièce aux échecs est le roi en vertu de son rôle dans le jeu, il semble que l'on doive effectivement en conclure que le roi a une signification au même sens auquel les signes dans une langue en ont une.

\section{Conclusion}

En résumé, j'ai noté dans la section 1 quelles difficultés soulève aux yeux de certains commentateurs le concept de jeu de langage. La principale était qu'un jeu de langage, même un jeu " simple » et « primitif » comme celui du constructeur, est supposé être complet (et indépendant des autres jeux) et devrait par conséquent constituer en lui-même un langage. En quel sens dire qu'il s'agit d'un langage ? J'ai essayé de l'expliquer dans la section 2 . Il y a langage, c'est-à-dire un emploi de signes doués de signification, à la condition que les signes aient un " usage ", c'est-à-dire qu' ils permettent comme dans l'exemple du jeu du constructeur de faire des énoncés jouant un rôle dans le contexte d'échanges humains. Comme l'écrit Wittgenstein dans De la certitude : «Nos paroles [nos activités linguistiques] reçoivent leur sens de nos autres actions ${ }^{84}$. " Le concept de jeu de langage, en effet, a entre autres l'utilité de montrer, au moins pour le cas de jeux simples, en quoi cela consiste pour un son ou une inscription que d'avoir une signification. La question qui se posait ensuite était celle de l'utilité du concept de jeu de langage pour une théorie des langues naturelles. Pour Wittgenstein, comme je l'ai indiqué dans la section 3, une langue naturelle est un «mélange" ou une "collection » d'un très grand nombre de jeux de langage (la plupart étant beaucoup plus complexes que le jeu du constructeur). La question qui se posait alors était : les énoncés d'une langue naturelle ont-ils une signification au même sens où les énoncés faits dans un jeu de langage simple en ont une ? J'ai traité cette question dans la section 4 . Il en est entre autres ressorti que nos jeux de langage ne sont pas pour la plupart «complets » et mutuellement « indépendants » comme l'est le jeu du constructeur. J'ai essayé pour terminer de répondre à des objections qui pouvaient être élevées contre la thèse que j'ai défendue d'après laquelle les Recherches contiennent une théorie du langage et de la signification ${ }^{85}$.

\section{Département de philosophie CEGEP de Saint-Hyacinthe}

84. L. Wittgenstein, Über Gewissheit, G. E. M. Anscombe et G. H. Von Wright (éd.), New-York, Harper and Row, 1969, § 229.

85. Les recherches qui ont mené à la rédaction de cet article ont été subventionnées par le FCAR. 